Article

\title{
Mediatization of Social Space and the Case of Uber Drivers
}

\author{
Ngai Keung Chan* and Lee Humphreys \\ Department of Communication, Cornell University, Ithaca, NY 14853, USA; E-Mails: nc478@cornell.edu (N.K.C.), \\ Imh13@cornell.edu (L.H.) \\ * Corresponding author
}

Submitted: 18 December 2017 | Accepted: 28 February 2018 | Published: 25 May 2018

\begin{abstract}
Digital data have become a form of "objectivation", which affect how we construct social knowledge and organize social space (Couldry \& Hepp, 2017). The workplace is one sphere that is increasingly datafied. This study explores how Uber drivers, a form of digitally-enabled service workers, contribute to the normalization of the social production of space through their interpretative practices of digital data in an online forum. Drawing on Uber's corporate discourse and an Uber driver online forum, we analyze two facets of the Uber app and drivers' mediated experiences: (1) the quantification and discipline of drivers' performance through Uber's rating system and (2) the coordination of spatial movement through location-related metrics. We argue that the underlying workings of the Uber app premediate expectations of service encounters and spatial movement. Uber drivers meanwhile develop practices which respond to and circumvent their own data contributions to the system. Drivers' practices, we argue, are largely in compliance with the calculative logics set by Uber. The article addresses implications of Uber drivers' practices for the reproduction of social space and power-relations in digitally-enabled service work and the gig economy.
\end{abstract}

\section{Keywords}

digitally-enabled service work; mediatization; mobile apps; ratings; social space; Uber; work practices

\section{Issue}

This article is part of the issue "Rethinking Media and Social Space", edited by André Jansson and Johan Lindell (Karlstad University, Sweden).

(C) 2018 by the authors; licensee Cogitatio (Lisbon, Portugal). This article is licensed under a Creative Commons Attribution 4.0 International License (CC BY).

\section{Introduction}

Datafication is the latest wave of mediatization, a wave that is deepening people's connectedness to data-driven infrastructures of communication-or what Couldry and Hepp (2017) call "deep mediatization". The ways that we make sense of reality, construct social knowledge and organize social space are intimately connected to digital data. The workplace is one sphere that is increasingly dataifed: not only do knowledge workers need to work with data and algorithms, but service workers are also increasingly required to interpret and use data in their daily work. The proliferation of digital and mobile technologies has rendered a variety of labor in the global digital economy-from data-entry workers to electronic workers to unpaid consumption labor-largely low paid or un- paid and invisible (Dyer-Witheford, 2015; Huws, 2014; Scholz, 2017). The expansion of gig economy is part of this broader context: inequality and precarity are deeply embedded in the gig economy (Chen, 2017; Schor \& Attwood-Charles, 2017; van Doorn, 2017). Against the backdrop of datafication and the gig economy, this study takes Uber as a case to explore how Uber drivers interpret the data generated through Uber mobile application (app) to mediate power-relations between the company, drivers, and riders. We use the term "digitallyenabled service workers" to refer to workers who interact with consumers virtually before meeting in the physical space. Like taxi drivers (Anderson, 2014; Davis, 1959) and other traditional service workers (Leidner, 1999), digitally-enabled service workers need to evaluate interactional contexts, and thus manage emotional dis- 
plays during service encounters. Scholars have studied the power-relations between service workers and consumers (e.g., Leidner, 1999; Lopez, 2010) and the instrumentality and socio-economic forces of performing affective or emotional labor (e.g., Duffy, 2016; Gregg, 2010; Hearn, 2010; Hochschild, 1983). Yet, central to the notion of digitally-enabled service workers is the temporal ordering of social interactions. The temporality is essential to the mediatization of social space in two important respects. First, data set expectations of social interactions. Second, data as a kind of social stock of knowledge (Couldry \& Hepp, 2017) may exercise control over drivers' work practices, whereas drivers may also develop practices to negotiate what counts as relevant "knowledge" in the gig economy.

An investigation into how Uber drivers interpret data helps to understand the production of social space because people access and use the Uber app with their mobile phones in the public space. We draw on Jansson's (2013) framework of mediatization and social space to explore the "transmedia textures" of Uber from the drivers' perspective. Echoing Humphreys' (2012) observations of mobile social networks, the use of Uber app connects drivers with riders who are not physically present, coordinates users' spatial movement, and catalogues metaspatial information about users of the app. As such, Uber drivers become a node within physical and datafied space. Uber drivers interact with riders in physical space and data generated through the app by continually sharing personal and locational information with other users and the company. Digital data and associated algorithms are socially constructed artifacts (Gillespie, 2014) that are built into drivers' work practices.

While much research has examined the governance by digital data in the workplace (e.g., Rosenblat \& Stark, 2016) and the ways that knowledge workers respond to dataifcation (e.g., Christin, 2017), this study explores digitally-enabled service workers' interpretative practice of data. Here, interpretative practice means the ways that Uber drivers articulate norms guiding their peers in engaging with data on an online forum where they build communication networks (Rosenblat, 2018). Such practices can contribute to the normalization of the social production of space (Jansson, 2013). We scrutinize how Uber's corporate discourse and Uber drivers' practices of knowledge sharing ascribe meanings to the data concerning physical space and their social interactions therein. This case study affords significant opportunities for thinking about the digitally-enabled service workers' labor experience and the socio-technical context where they interact with the platform, the company, and riders.

\section{Literature Review}

\subsection{Mediatization and Social Space}

Theoretically, we explore how Uber contributes to the production of social space. Jansson's (2013) framework on the mediatization of social space is especially helpful in conceptualizing Uber and the mediatization of social space. He uses Lefebvre's (1991) triadic understanding for social space, that is, space is produced through perception, conception, and lived practice. Jansson (2013) mediatizes this triadic frame to account for the contemporary transmedia environment we live in today. He argues that perceived space "directs our attention towards the more material, sensuous dimensions of the media" (p. 282), that is, in a transmedia world, perceived space takes on material indispensability and adaptation. Within the context of this study, we will examine how Uber drivers perceive space through their interactions with the Uber app. According to Jansson, conceived space can be best understood through the premediation of experience and expectations. This suggests an attention to the ways that the experience of space is premediated for drivers (as well as riders). Important to Lefebvre's notion of conceived space are power differentials. That is, some actors have more power than others in defining and shaping what space is and how it is used (Harvey, 1990). For example, Uber has its own navigation system that is directly embedded within the Uber driver app. This navigation system is an example of the kinds of premediated, conceived spaces which are essential to the infrastructure of Uber as both an app and a company. It essentially premediates the coordination of driver and rider such that one must use the app to summon a driver.

Lastly, and of great relevance to this article, is Jansson's (2013) normalization of social practice. Most similar to Lefebvre's (1991) lived experience, this concept helps to understand how Uber drivers seek to influence the production of space through their appropriation of Uber app, its various features and various other media used in concert. Jansson draws our attention to the normalization of social practice, that is, how space is produced through the "norms, conventions and expectations" of everyday life (2013, p. 285). Therefore, to study not just spatial practice, but it's normalization, we empirically examine as one of our data sources an online drivers' forum where such normalization is explicitly articulated rather than inferred from ethnographic observation.

The question of the mediatization of social space within the case of Uber requires not only definitions of what we mean social space but mechanisms within mediatization that more specifically contribute to the study of Uber. In this case, datafication.

\subsection{The Politics of Data}

Data and associated algorithms can be inherently political artifacts because they can produce and objectify certain logics of social knowledge (Couldry \& Hepp, 2017; Gillespie, 2014). Gillespie (2014) has argued algorithms evaluate and recommend "relevant" information to the users, based on their implicit assumptions and values about what counts as "legitimate" knowledge. Because algorithms cannot achieve its ends without users' prac- 
tices, we should examine the "entanglement between algorithms put into practice and the social tactics of users who take them up" in broader socio-technical contexts (Gillespie, 2014, p. 183). An examination of entanglement with practice has to do with the interpretative agency of users in negotiating meanings of data. Couldry and Hepp (2017) have explicated that datafication is an emerging wave of mediatization because data become a form of "objectivation" that affects how we construct social reality, though people may not be aware of the automated processes of classifying and categorizing the data. They argue data can be translated into social practices through the organization of social space, time, self, collectivities, and social order.

This study focuses on the intersection of data and social production of space. The Uber app not only categorizes and segregates physical space through algorithms but also becomes the means to coordinate drivers' and riders' spatial movement, trace their spatially relevant information, and encounter other users. Uber's rating system also produces "calculated publics" (Gillespie, 2014) by categorizing drivers and riders into different groups on a five-star scale. As such, the data generated via Uber app, which embody Uber's implicit assumptions about social knowledge, may affect drivers' interpretation of space and interactions therein. But Uber drivers must consider how they react to data. Do they consider the data as a form of social knowledge that shapes their interpretation of physical space and social interactions with riders? How do drivers interpret the data for their purposes? This study may provide a glimpse of these issues and contribute to the literature on the mediatization of social space.

\subsection{Datafication of the Workplace and Workers' Autonomy}

The datafication of the workplace involves processes through which employers and workers negotiate what counts as "knowledge" in making work-related decisions (Stark, 2009). Braverman (1974) has highlighted a complex relationship between workers' skills, knowledge, job autonomy and managerial control. Managerial control is executed through the abstraction of and "monopoly over knowledge" (Braverman, 1974, p. 82). This form of control concentrates the ownership of production knowledge in the hands of employers and excludes workers from this process. Braverman's work offers precious insights into the labor power of knowledge and the dynamics of control and resistance. Recently, corporations deploy data-driven technologies to restructure labor process through the abstraction of data and knowledge (Levy, 2015). Therefore, an investigation into the relationship between labor process and datafication as knowledge production can help to understand power dynamics in the digital economy.

Existing research largely have examined how knowledge workers-for example, journalists (Anderson, 2011;
Carlson, 2017; Christin, 2017), legal experts (Christin, 2017), medical professionals (Maiers, 2017) and educators (Sauder \& Espeland, 2009)-respond to the institutional demand for algorithmic decision-making. An underlying premise is that algorithms can make more "objective" and "rational" judgment that humans, from the management's perspective (Carlson, 2017; Christin, 2017). Data become a form of quantifiable knowledge that may change workers' decision-making process. For instance, managers ask journalists to make news decisions based on web traffic statistics (Anderson, 2011) and use algorithms to produce, prioritize, and recommend news to their readers (Carlson, 2017). Yet, there are discrepancies between organizational policies and workers' actual practices. Christin (2017) found that journalists and legal professionals develop buffering strategies to resist such technologies. The workers may simply ignore the metrics generated by the technologies, selectively manipulate the data for their goals, and criticize the validity of the data.

Organizational culture and professional agency can affect how people interpret data and knowledge within their professions. For example, Maiers (2017) found a tension between quantifiable metrics created by Horizon, a data-driven medical technology, and clinicians' tacit knowledge. Although clinicians recognize the value of Horizon, they do not take the data as the only legitimate knowledge. Rather, they may engage in "conditioned reading", by tracking both the data and other indicators of patient health in the decision-making process. "Conditioned reading" of data is possible in organizational contexts which recognize the agency of workers to draw on their expertise. As such, knowledge workers' professional expertise becomes a vital source of job autonomy.

White collar workers, however, may be very different from low-wage service workers in labor conditions and experiences. Employers reportedly discipline low-wage workers through a system of scientific management (Ball, 2010; Braverman, 1974), refractive surveillance (Levy \& Barocas, 2018) and automated surveillance (Levy, 2015; Moore, Upchurch, \& Whittaker, 2018; Rosenblat \& Stark, 2016). For example, Levy (2015) found that truck firms used real-time fleet management systems to create abstract data streams to constantly monitor drivers' work schedule, geolocation, and duty status. Such managerial practice considered truck drivers as a mere data point in the technological system, thus allowing the management to control workers' spatial movement in time. Hence, lowwage service workers have limited job autonomy (van Doorn, 2017). This raises the questions of how service workers may imagine and respond to the datafication.

We argue that Braverman's (1974) discussion of managerial control as the abstraction and monopoly of knowledge is particularly relevant to datafication, labor process and power dynamics in service work. Recent studies have complicated the emotional labor process by recognizing the labor subjectivity and struggles to 
perform emotion work contingent upon organizational and situational demands and worker-consumer relationship in service work (Bolton, 2010; Lopez, 2010). In the context of Uber, we should consider who has more power to define the norms of service interactions. Additionally, Levy and Barocas (2018) have developed the framework of refractive surveillance to situate consumer surveillance and worker control relationally, particularly in the low-wage retail workplace. Corporations deploy consumer data, such as customer in-store experiences, to make decisions as to the management of employees work hours and scheduling. It helps to understand how Uber's consumer-sourced rating system may exercise power over drivers. Drawing insights from these studies, we examine how digital data shape Uber drivers' interpretative practices and the labor process in the age of deep mediatization.

\subsection{Uber Drivers in the Gig Economy}

Over the last decade, there has been a skyrocketing growth of the so-called "gig" or "sharing" economy that deploys algorithmic technologies to manage and organize work (Scholz, 2017). The on-demand platforms, such as Uber, consider both service providers and consumers as users of their services, which allow the companies to distance themselves from an employment relationship with service providers (e.g., Uber drivers) and associated obligations (van Doorn, 2017). The construction of platforms may also aggravate class, racial and gender inequalities during service encounters (Schor \& AttwoodCharles, 2017) and result in workplace discrimination (Rosenblat, Levy, Barocas, \& Hwang, 2017). Situating in the wider context of precarious workforce, the gig economy can be seen "an opportunity to increase labor control while externalizing risks onto contractors and customers" (Schor \& Attwood-Charles, 2017, p. 7). Similarly, Fleming (2017) has argued that there is a "radical responsibilization" of work, which is intimately connected with neoliberal discourse that emphasizes individual responsibility and choice. The discourse may normalize gig workers' precarious experiences. Moreover, despite the platform owners' glamorization of flexibility of gig workers, workers are subject to expansive socio-technical control (van Doorn, 2017) through information asymmetries (Rosenblat \& Stark, 2016; Shapiro, 2017). The platforms can create information asymmetries through selective display of relevant work-related information. In a study of on-demand courier services, Shapiro (2017) found that the companies remove locational information in the app interface, which delimits gig workers' abilities to decide whether to accept job orders. Yet, Shapiro notes that gig workers' intuitions and experiences might affect their interpretation of data in making decisions. Therefore, it is crucial to understand how gig workers may normalize and entangle with the calculative logics of algorithms.

Studies on Uber drivers have extended critiques of labor practices in the gig economy, by examining how
Uber app may facilitate automated algorithmic management of labor (Gloss, McGregor, \& Brown, 2016; Lee, Kusbit, Metsky, \& Dabbish, 2015; Rosenblat \& Stark, 2016). The app's navigation and rating systems enable constant surveillance of drivers. Because the potentially biased ratings determine drivers' employment opportunities (Rosenblat et al., 2017), the rating system lead drivers to perform emotional labor for an exchange of a good rating (Gloss et al., 2016; Raval \& Dourish, 2016; Rosenblat \& Stark, 2016). Due to the information asymmetries between the company and drivers, drivers often expressed frustration with the data generated via Uber app, which necessitate learning how to interpret such data (Gloss et al., 2016; Malin \& Chandler, 2017; Raval \& Dourish, 2016; Rosenblat \& Stark, 2016).

These studies provide valuable insights into the labor control and practices in the gig economy and the governing role of Uber app, but few of them explicated how the service encounters are mediatizated through the datafication of drivers' performance and physical location. Therefore, we examine how drivers articulate norms of interpreting the data generated via Uber app to construct social space and shape interactions between drivers, riders, and the platform itself.

\section{Research Context and Methods}

Uber, founded in 2009, is one of the largest ride-hailing service companies. In 2017, there were 3 million active drivers globally (Bhuiyan, 2018). Using Uber app, Uber drivers can provide ride-hailing services to other users who request a ride. The app monitors users' locations and performance metrics in real-time contexts. Echoing Bowker and Star's (1999) discussion of classification systems, we argue that the data generated and exhibited through the app are always incomplete and conduct invisible work to organize drivers' work in social space. This study focuses on two facets of drivers' mediated experiences, namely the rating system and the navigation system.

Methodologically, the construction of algorithmic systems is largely black boxed because platform owners prohibit individuals from accessing the design process and the algorithms are always evolving. A discursive analysis of platform owner' documents and different actors' interpretative practices is a useful strategy to understand algorithmic systems (Kitchin, 2017). Specifically, the materials presented in this article draw on (1) Uber's website and (2) online forum for Uber drivers. We analyzed the company documents concerning the rating system and navigation system to understand how Uber has framed the two systems and intended users' action. We also draw on UberPeople, one of the largest online Uber driver forums. By December 2017, the online forum had about 110,000 members and 198,000 discussion posts, where drivers discuss the strategies for economic success. We do not intend to generalize drivers' practices of knowledge sharing on this forum to the ac- 
tual work practices of the whole population of Uber drivers. Yet, because the forum represents a place where drivers share work-related knowledge (Rosenblat, 2018), our analysis of the forum discussion may reveal how some drivers articulate norms and expectations of digital data through their practices of sharing peer-to-peer knowledge. We conducted a purposive sampling (Coyne, 1997) of discussion threads, specifically focusing on two of the thirteen main discussions threads on the site: "Ratings" and "Advice". We analyzed the most-viewed post on the "Ratings" thread (i.e., more than 100,000 views) to explore how drivers interpret and manage the rating system. Within "Advice", we examined the "Beginner Advice" posted by the moderator of this forum, which may help us to understand how drivers articulate their knowledge about the data with other drivers. A comparison between Uber's documents and drivers' posts reveals discrepancies between Uber's policy and drivers' interpretative practices as well as the negotiation of legitimate knowledge around service encounters.

\section{The Management of an "Imperfect" System}

As an algorithmic labor management system (Rosenblat \& Stark, 2016), Uber app is an "imperfect" system because it can never fully control drivers' performance. Based on the materials we collected, Uber and drivers negotiate the meanings of the data generated via the rating system and the aggregated location-related metrics. There are two recurrent themes surrounding the use of Uber app and datafication: (1) the quantification and discipline of drivers' performance and (2) the coordination of spatial movement. Examples within each theme demonstrate how the digital data mediate the social production of space and the power-relations between Uber and drivers.

\subsection{The Quantification and Discipline of Drivers' Performance}

Uber's rating system allows drivers and riders to evaluate one another's performance after each trip on a generic 5 -point star system. One's overall rating is an average of the ratings she or he got from the last 500 trips. Uber drivers are required to rate riders' performance after each trip, whereas riders can decide whether they rate their drivers. The consumer-sourced rating system offers a mechanism for Uber to measure drivers' performance and legitimize their decisions on who can continue working on the platform. However, Uber enforces a stricter regulation on drivers than riders because Uber does not deactivate riders' account even they get a poor rating.

Uber's rating system can be seen as disciplinary practices that exercise power through surveillance and normalization (Sauder \& Espeland, 2009). Uber not only continuously monitors drivers' performance through the real-time rating system (Rosenblat \& Stark, 2016) but also disciplines drivers to keep track of their quanti- fied performance. The rating system normalizes such discipline by standardizing the "ideal" ride-hailing experiences and creating the normative hierarchy (Sauder \& Espeland, 2009). The rating system situates drivers' performance relationally: a driver's rating reflects not only one's performance but also the extent to which one's performance conforms to the standard norms of "ideal" performance held by other drivers. Because the meanings of one's numeric star rating are abstract, Uber sent a guide to the drivers to explain the methods of calculating ratings, the goals of using the rating system, and strategies for getting a good rating in 2014. This official guide documents how drivers should perform during the ride. The guide explained, "Your [drivers'] average rating is the first thing that Uber uses when evaluating your [drivers'] partnership and the system" (Uber, 2014, p. 2). Uber may deactivate the "lowest-quality drivers" to protect "the quality of the Uber system as a whole" and ensure that riders can enjoy an efficient and safe ride-hailing service (Uber, 2014, p. 3). This document also provides some guidelines for getting a high rating. Specifically, drivers should "offer riders bottled water, snacks, gum, and cell phone chargers" and have a clean car (Uber, 2014 , p. 8). The drivers should also perform emotional labor by dressing professionally and being "friendly and positive, regardless of the rider's attitude" (Uber, 2014, p. 10). In 2017, Uber's website included these suggestions and added that drivers should "keep the conversation polite, professional, and respectful" and be "sociable" (Uber Help, 2017).

Uber's suggestions are relevant to the mediatization of social interactions through datafication. The aggregated consumer-sourced ratings become the mediated knowledge that provides personalized recommendations to drivers and Uber about drivers' work performance. Because drivers' rating determines their employment opportunities, they have to discipline their performance to get high ratings. The Uber's guideline also envisions that drivers' interactions with riders should facilitate an efficient, safe, and communal trip. The rating system thus becomes a form of "feeling rules" (Hochschild, 1983; Leidner, 1999) that standardize and control how drivers should interact with riders in both mediated and face-to-face communication. The system also structures drivers' feelings and monetizes affective relationships into a form of digital reputation (Hearn, 2010). The instrumentality of affective relationships is not new; for example, taxi drivers have long managed their affective relationships with passengers to solicit tips (Davis, 1959). The difference is that Uber possesses much more information about service encounters than drivers and can use the information to script drivers' performance through the deployment of data-driven technologies (Levy, 2015). Hence, drivers have limited degree of autonomy in deciding their performance during service encounters.

Indeed, Uber drivers may socialize themselves to the standard created by Uber's rating system. On UberPeople, there are heated discussions on the tactics of get- 
ting a good rating. Participants consider drivers as vulnerable subjects because riders can unreasonably give them a low rating and there is no formal procedure for drivers to complain about the ratings. The uncertainty and anxiety created by the rating system are related to the data's disciplinary power because drivers have limited power to manage the evaluative process (Sauder \& Espeland, 2009). Thus, drivers attempt to comply with the expectations created by the system. Specifically, some participants recommended others to follow Uber's suggestions to dress professionally and learn how to communicate with riders in a friendly way. In a discussion post with more than 600 replies, participants suggested that "if there are more than one passenger in the car, be sure to engage them all equally in conversation". When more than one rider enters the car, drivers cannot know who will be rating them. Engaging all passengers mitigates this uncertainty. Drivers should also know when they should be "quiet" or "chatty" by observing riders' performance and body language. It also means that drivers should not talk about sensitive topics such as politics because it may negatively affect the rating. Like Uber's suggestions, participants recommended that drivers should offer bottled water and phone chargers to riders. These suggestions may normalize the meanings Uber scripts into its rating system; that is, drivers conform to the algorithmic power by learning to be "professional" and "sociable" service workers.

Unlike professional workers (Christin, 2017), drivers work in a highly constrained environment where they cannot simply ignore data and have limited autonomy to interpret data. The forum provides drivers with an opportunity to denounce the limited transparency and unfairness of Uber's rating system. On UberPeople, participants suggested that there are various reasons for a poor rating, based on their work experience. They explained that young people, nighttime riders, and the drunk are more likely to give low ratings. The working time and locations are vital to their ratings because if drivers mostly work in a "party city at night", they tend to get a poor rating. One participant stated:

I intend to be a full driver and make a lot of money for this company and don't feel drivers should have to live in fear of losing an account over the actions of the intoxicated. Please look at the rating system and allow it to give us a chance to grow rather than live in fear.

The consequence of getting a poor rating is the prohibition of working on Uber. The rating system thus datafies the social interaction as well as the physical vehicular space, which can contribute to a drivers' perpetual state of fear. Because drivers cannot continue working through Uber if their rating is below about 4.6/5.0, one to four stars is regarded as failing grade. Drivers contended that Uber should educate riders about the rating system. Other drivers stated that the rating system is po- tentially biased because the system elevates riders' evaluation over drivers' unique work experiences. Yet, riders may have implicit biases when they evaluate drivers' performance; for example, they may tend to give a low rating if the driver already has a poor rating. The imperfect navigation system can also result in an unsatisfactory rating. Because drivers do not control the rating system by which they are evaluated, forum participants closely attend to the details of the system. This in turn reveals how they internalize the system as a form of discipline.

Another strategy of managing the rating system is "gaming", which is defined as "cynical efforts to manipulate the rankings data without addressing the underlying condition that is the target of measurement" (Sauder \& Espeland, 2009, p. 76). For Uber drivers, a gaming strategy is not accepting certain riders' request, based on riders' location and performance metrics. When drivers receive a ride request, the app will show the time distance between drivers' location and the pick-up location. Participants on UberPeople proposed that drivers should not accept a ride that is more than eight minutes away from their current location because when riders wait for a long time, they are more likely to rate the drivers low. Moreover, forum participants contended that drivers should not accept riders with a rating below 4.7/5.0 because these riders are usually "troublesome" and less likely to tip the drivers. Uber never suggests that drivers can select a rider based on the ratings or location-related data. Yet, drivers appropriate the system to protect their employment opportunities. Although drivers are vocal in their criticism of the rating system, they nevertheless legitimize the system by ascribing social meanings (e.g., troublesome) to riders with a substandard rating. In all, the rating system becomes a datafied form of discipline that shapes how drivers interact with the platform and riders. Simultaneously drivers develop interpretative practices that utilize the data to sustain their livelihood.

\subsection{The Coordination of Spatial Movement}

One common way for drivers to use the Uber app to connect with place was to use the navigation system. The app includes the GPS navigation system, but drivers can also use other systems to navigate their route such as Google Maps. The navigation system not only is a representation of the city but also creates premediated socio-spatial relations between drivers and riders. As Uber claims, "navigation means more than just getting from point $A$ to point $B$. For example, upon arriving at a pickup point, drivers then have to find their riders - right down to what side of the street they're on" (Uber Newsroom, 2017). Participants on UberPeople stated that riders might give a low rating to the driver who has a bad navigation, though Uber has recently attempted to address this issue. From drivers' perspective, there are various reasons that can affect the accuracy of the navigation system. One example is the driver's physical location: 
drivers may have inaccurate GPS signals when driving in the central business district or through tunnels. Hence, participants suggested that drivers should have knowledge about the city and only drive in the places where they are familiar with, as they cannot always rely on the navigation system. Additionally, one major intent for drivers to use the navigation system is to communicate and coordinate with riders. This coordination process involves riders' input of the pick-up location. Nonetheless, participants found that riders might not enter the most accurate address, especially in the central business district or places with multiple entrances. The "Beginner Advice" on the forum made a distinction between "safe" and "unsafe" places when discussing the navigation system. The "safe" places mean the location that can easily identify the rider and stop the car, whereas examples of "unsafe" places include the places located at the major intersection with traffic congestion. To manage the imperfect navigation system, drivers did turn urban spaces into meaningful places where they meet riders.

Another set of practices concerns the surge pricing feature. This shows "real-time" demand and supply of ride-hailing services in the city and determines when drivers can get higher ride rates due to higher demand. By offering higher rates to drivers working in the surge area, the mediated location awareness encourages drivers to work in particular places with high demand. Rosenblat and Stark (2016) have argued that surge pricing shows the inaccurate predictive demand of the services, which can undermine drivers' autonomy. On the forum, drivers noted that surge price is based on riders' rather than drivers' location. Even when the drivers' app shows surge pricing, drivers need to pay attention to the popup notification regarding the particular ride request because only that notification will determine the actual ride rate. Therefore, some participants suggested that drivers should ignore the surge pricing because "surges last for minutes and there is no guarantee to get a surge job". Some even complaining that "Uber creates fake surges".

Moreover, drivers tried to leverage the data from the surge pricing maps for their benefit. For example, drivers could attend to the major events in the city such as concerts to predict and identify the surge zone before the app shows the increased surge pricing. As one blogger explained:

What I found that works best is to stay at the outskirts of the surge zone or to identify a secondary surge zone....What I found was that I lost a lot of time looking for riders, and then I would have to cancel. By that time, the surge was over and now I just lost time and money at this big event. A better strategy is to go a little before the event is over and after the big crowd is gone. It is easier to move in and out of the area and you can get multiple rides as opposed to one big ride. Once the surge is gone, I go back to the area because some people hang out at the bars until they close. (Castillo, 2017)
Overall, drivers recommended acquiring knowledge about the city (e.g., the location and time of the major events) if they want to utilize the surge pricing to maximize profit. However, distrust in the mapping system, again, reveals the complex ways drivers must manage digital data in their service work.

\subsection{Summary and Discussion of the Findings}

Grounded in the premise that "automated processes of data-processing are deeply embedded in" Uber drivers' daily work (Couldry \& Hepp, 2017, p. 126), we argue the Uber app contributes to the mediatization of social space in several important ways. Algorithms construct a basis for decision-making, often based on the seemingly "neutral" adaptive statistical techniques that classify the relationship between digital data and users' feedback and structure the circulation of information (Rieder, 2017). Although our goal is not to study the underlying statistical practices that the Uber app relies upon, it is vital to note that the selection of certain calculative procedures and the ways of classifying data are deeply social decisions. We suggest that the underlying workings of the app premediate expectations of service encounters, particularly the ways that drivers and riders should perform. We have offered a preliminary assessment of Uber's discourse and drivers' practices surrounding the rating system and location-related metrics. Because of the navigation system and surge pricing, drivers develop practices which respond to and circumvent their own data contributions to the system. The sharing of such practices on UberPeople contributes to the normalization of the social production of space.

Based on an analysis of UberPeople, we argue Uber drivers have a distinct algorithmic imaginary, that is, "ways of thinking about what algorithms are, what they should be, how they function, and what these imaginations in turn make possible" (Bucker, 2017, p. 40). Our use of the term "imaginary" aims to highlight digital data are internalized and forms part of drivers' understanding of the repertoire of everyday work practices. Uber drivers are aware that digital data, such as their ratings, can affect their employment opportunities. Therefore, drivers mobilize their algorithmic imaginary to criticize the opaque methods of calculating and using the performance metrics and location-related data. They nonetheless have limited ability to change the system, due to the information and power asymmetries between the company and drivers. Workers may learn to negotiate with algorithmic labor management, as they familiarize with the app (Shapiro, 2017). On UberPeople, drivers develop strategies to adapt their work practices to the available data to keep their jobs and maximize their earnings. Drivers also attempt to validate riders' performance through quantifiable metrics, though they meanwhile denounce the inaccuracy of the rating system. In other words, drivers take digital data as a kind of social knowledge and normalize the idea that they have to learn to live with digital data. 
There are tensions between Uber's discourse and drivers' practices surrounding the Uber app, which contribute to the mediatization of social space and interactions therein. Uber's discourse regards the data generated through the navigation and rating systems as "objective" knowledge that helps drivers to make informed decisions. The location-related metrics, including the data generated via the navigation system and surge pricing, not only signify the location of drivers but also visualize where riders are. The digital map is thus a representation of the city, based on the algorithmic calculation of the consumer demand. The Uber app becomes a datafied space that provides work-related knowledge about the city and enables social coordination across "calculated" spaces. Yet, the accuracy of the navigation system is determined by drivers' physical location. Therefore, drivers need to manage the imperfect system by acquiring the knowledge about the city and attaching meanings, such as familiarity and safety, to physical locations where they drive. In other words, the premediation of drivers' experiences, the material indispensability of the app itself, and the normalization of drivers' social practice converge to produce social space in a datafied and mediatized world.

Additionally, the rating system is an imperfect system that standardizes drivers' service interactions and ride-hailing experiences. While Uber attempts to control drivers' work performance, drivers simultaneously develop their norms of interpreting the ratings through practices of knowledge sharing on the forum. This is not to suggest that drivers' practices are resistance to Uber's managerial control, but to demonstrate how drivers may normalize the managerial control and develop reactive strategies to evade punishment. To qualify as an act of resistance, the resister's intention of undermining powerrelations should be recognized by the opposition and bystanders (Hollander \& Einwohner, 2004). In this case, drivers' interpretative practices are largely in compliance with the calculative logics established by Uber. Nonetheless, we highlight the interpretative flexibility (Pinch \& Bijker, 1984) of the rating system by attending to how Uber and drivers ascribe meanings to the system. The rating system carries not only managerial control over the drivers but also drivers' interpretation of riders. The tensions outlined above suggest that digitally-enabled service work, analogous to expert fields (Christin, 2017), is a complex avenue where managerial discourses and workers' actual practices may differ with one another.

Our preliminary assessments of the management of the imperfect system can provide insights into the politics of data. In the workplace, digital data and associated algorithmic systems embody the management's prescriptive assumptions about the "relevant" knowledge (Gillespie, 2014), but they can never fulfill all the needs of workers. From workers' perspective, digital data as social knowledge are always imperfect yet can be used to help manage risk. By attending to workers' practices that manage such imperfectness, we may be able to the power dynamics mediated by data.

\section{Conclusion}

This study makes three contributions to the literature around datafication of the workplace. First, by exploring how Uber drivers interpret the performance and location-related metrics, we demonstrate how digital data can construct and set expectations for social interactions. In the wave of datafication, data constitute media space of social contestation (Couldry \& Hepp, 2017). Our study shows that the Uber drivers negotiate with the premediation of spatial experience and articulate norms of social interaction in the datafied space. Second, we highlight that digitally-enabled service work is a vital site of encountering and contesting the datafication. Recent studies have drawn our attention to the importance of "context" when considering the presumed and actual impacts of algorithms (Bucker, 2017; Christin, 2017). We suggest the term digitally-enabled service workers to better incorporate the processes through which digital data set service workers' expectations of social interactions and mediate the power dynamics in service work. In keeping with previous research (Rosenblat \& Stark, 2016), we find that Uber deploys information asymmetries to delimit drivers' decision-making capabilities. Drivers develop a distinct algorithmic imaginary based on the calculative logics of digital data and their peerto-peer knowledge about the app and work experiences. Uber drivers are just one group of gig workers in the precarious and datafied workforce. Further research should explore how organizational and social differences between professional workers and service workers shape their algorithmic imaginaries and the power-relations in the workplace. Moreover, it is possible that forum participants are more familiar with the Uber app than others. Future studies should explicate whether drivers' interpretative practices around digital data vary in their skills and socio-cultural background. Third, extending critiques over labor practices in the gig economy (Chen, 2017; Rosenblat \& Stark, 2016; van Doorn, 2017), this study highlights how data become a form of social knowledge that can favor the company to concentrate the ownership of actionable information and exercise power over workers. Though drivers' reactive strategies are far from resistance to Uber's managerial control, the online forum seems to represent a potential place for workers to create and circulate "worker-generated knowledge" (Chen, 2017) that may empower drivers to make their work experiences visible and cultivate their own workplace culture (Rosenblat, 2018). While the on-demand business model "currently dominates corporate 'future of work' imaginaries" (van Doorn, 2017, p. 908), it is vital to explore how workers can possibly counteract algorithmic power of corporate platforms and build a socially fairer digital economy (e.g., platform cooperativism, see Scholz, 2017). There are signs that some gig workers engage in digital activism that resists platform algorithms (e.g., Chen, 2017). Future work should examine workers' contingent labor conditions in different forms of gig 
work and broader socio-technical contexts that can empower workers.

\section{Acknowledgments}

We are grateful to the editors and the three anonymous reviewers for their insightful suggestions and comments.

\section{Conflict of Interests}

The authors declare no conflict of interests.

\section{References}

Anderson, C. (2011). Between creative and quantified audiences: Web metrics and changing patterns of newswork in local US newsrooms. Journalism, 12(5), 550-566.

Anderson, D. N. (2014). The taxicab-hailing encounter: The politics of gesture in the interaction order. Semiotica, 202, 609-629.

Ball, K. (2010). Workplace surveillance: An overview. Labor History, 51(1), 87-106.

Bhuiyan, J. (2018, January 5). Uber powered four billion rides in 2017. It wants to do more-and cheaper-in 2018. Recode. Retrieved from https://www.recode. net/2018/1/5/16854714/uber-four-billion-rides-coo -barney-harford-2018-cut-costs-customer-service

Bolton, S. (2010). Old ambiguities and new developments: Exploring the emotional labour process. In P. Thompson \& C. Smith (Eds.), Working life: Renewing labour process analysis (pp. 205-222). London: Palgrave.

Bowker, G. C., \& Star, S. L. (1999). Sorting things out: Classification and its consequences. Cambridge, MA: MIT Press.

Braverman, H. (1974). Labor and monopoly capital: The degradation of work in the twentieth century. New York, NY: Monthly Review Press.

Bucker, T. (2017). The algorithmic imaginary: Exploring the ordinary effects of Facebook algorithms. Information, Communication \& Society, 20(1), 30-44.

Carlson, M. (2017). Automating judgment? Algorithmic judgment, news knowledge, and journalistic professionalism. New Media \& Society. doi:10.1177/ 1461444817706684

Castillo, A. (2017, December 1). Why you shouldn't chase the surge: And other driving strategies. The RideShare Guy. Retrieved from https://theride shareguy.com/why-you-shouldnt-chase-the-surgeand-other-driving-strategies

Chen, J. Y. (2017). Thrown under the bus and outrunning it! The logic of Didi and taxi drivers' labour and activism in the on-demand economy. New Media \& Society. doi:10.1177/1461444817729149

Christin, A. (2017). Algorithms in practice: Comparing web journalism and criminal justice. Big Data \& Society, 4(2), 1-14.
Couldry, N., \& Hepp, A. (2017). The mediated construction of reality. Cambridge: Polity.

Coyne, I. T. (1997). Sampling in qualitative research. Purposeful and theoretical sampling; merging or clear boundaries? Journal of Advanced Nursing, 26(3), 623-630.

Davis, F. (1959). The cabdriver and his fare: Facets of a fleeting relationship. American Journal of Sociology, 65(2), 158-165.

Duffy, B. (2016). The romance of work: Gender and aspirational labour in the digital culture industries. International Journal of Cultural Studies, 19(4), 441-457.

Dyer-Witheford, N. (2015). Cyber-proletariat: Global labour in the digital vortex. London: Pluto Press.

Fleming, P. (2017). The human capital hoax: Work, debt and insecurity in the era of Uberization. Organization Studies, 38(5), 691-709.

Gillespie, T. (2014). The relevance of algorithms. In T. Gillespie, P. Boczkowski, \& K. Foot (Eds.), Media technologies: Essays on communication, materiality, and society (pp. 167-194). Cambridge, MA: MIT Press.

Gloss, M., McGregor, M., \& Brown, B. (2016). Designing for labour: Uber and the on-demand mobile workforce. In J. Kaye \& A. Druin (Eds.), CHI '16 proceedings of the $2016 \mathrm{CHI}$ conference on human factors in computing systems (pp. 1632-1643). New York, NY: ACM.

Gregg, M. (2010). On Friday night drinks: Workplace affects in the age of the cubicle. In M. Gregg \& G. J. Seigworth (Eds.), The affect theory reader (pp. 250-268). Durham, NC: Duke University Press.

Harvey, D. (1990). The condition of postmodernity: An enquiry into the origins of cultural change. Malden, MA: Blackwell.

Hearn, A. (2010). Structuring feeling: Web 2.0, online ranking and rating, and the digital 'reputation' economy. Ephemera: Theory \& Politics in Organization, 10(3/4), 421-438.

Hochschild, A. (1983). The managed heart: Commercialization of human feeling. Berkeley, CA: University of California Press.

Hollander, J. A., \& Einwohner, R. L. (2004). Conceptualizing resistance. Sociological Forum, 19(4), 533-554.

Humphreys, L. (2012). Connecting, coordinating, cataloguing: Communicative practices on mobile social networks. Journal of Broadcasting \& Electronic Media, 56(4), 494-510.

Huws, U. (2014). Labor in the global digital economy: The cybertariat comes of age. New York, NY: Monthly Review Press.

Jansson, A. (2013). Mediatization and social space: Reconstructing mediatization for the transmedia age. Communication Theory, 23(3), 279-296.

Kitchin, R. (2017). Thinking critically about and researching algorithms. Information, Communication and Society, 20(1), 14-29.

Lee, M. K., Kusbit, D., Metsky, E., \& Dabbish, L. (2015). Working with machines: The impact of algorithmic and data-driven management on human workers. In B. 
Begole \& J. Kim (Eds.), CHI '15 proceedings of the 33rd annual ACM conference on human factors in computing systems (pp. 1603-1612). New York, NY: ACM.

Lefebvre, H. (1991). The production of space (D. Nicholson-Smith, Trans.). Oxford: Blackwell.

Leidner, R. (1999). Emotional labor in service work. The Annals of the American Academy of Political and Social Science, 561(1), 81-95.

Levy, K. E. C. (2015). The contexts of control: Information, power, and truck-driving work. The Information Society, 31(2), 160-174.

Levy, K. E. C., \& Barocas, C. (2018). Refractive surveillance: Monitoring customers to manage workers. International Journal of Communication, 12, 1166-1188.

Lopez, S. H. (2010). Workers, managers, and customers: Triangles of power in work communities. Work and Occupations, 37(3), 251-271.

Maiers, C. (2017). Analytics in action: Users and predictive data in the neonatal intensive care unit. Information, Communication and Society, 20(6), 915-929.

Malin, B. J., \& Chandler, C. (2017). Free to work anxiously: Splintering precarity among drivers for Uber and Lyft. Communication, Culture and Critique, 10(2), 382-400.

Moore, P. V, Upchurch, M., \& Whittaker, X. (Eds.). (2018). Human and machines at work: Monitoring, surveillance and automation in contemporary capitalism. Basingstoke, UK: Palgrave Macmillan.

Pinch, T., \& Bijker, W. (1984). The social construction of facts and artefacts: Or how the sociology of science and the sociology of technology might benefit from each other. Social Studies of Science, 14(3), 399-441.

Raval, N., \& Dourish, P. (2016). Standing out from the crowd: Emotional labor, body labor, and temporal labor in ridesharing. In D. Gergle \& M. R. Morris (Eds.), CSCW'16 proceedings of the 19th ACM conference on computer-supported cooperative work \& social computing (pp. 97-107). New York, NY: ACM.

Rieder, B. (2017). Scrutinizing an algorithmic technique: The Bayes classifier as interested reading of reality. Information, Communication \& Society, 20(1), 100-117.
Rosenblat, A. (2018, January 9). The network Uber drivers built. Fast Company. Retrieved from https:// www.fastcompany.com/40501439/the-network-uber -drivers-built

Rosenblat, A., Levy, K. E. C., Barocas, S., \& Hwang, T. (2017). Discriminating tastes: Uber's customer ratings as vehicles for workplace discrimination. Policy \& Internet, 9(3), 256-279.

Rosenblat, A., \& Stark, L. (2016). Algorithmic labor and information asymmetries: A case study of Uber's drivers. International Journal of Communication, 10, 3758-3784.

Sauder, M., \& Espeland, W. N. (2009). The discipline of rankings: Tight coupling and organizational change. American Sociological Review, 74(1), 63-82.

Scholz, T. (2017). Uberworked and underpaid: How workers are disrupting the digital economy. Cambridge: Polity.

Schor, J. B., \& Attwood-Charles, W. (2017). The "sharing" economy: Labor, inequality, and social connection on for-profit platforms. Sociology Compass, 11(8), 1-16.

Shapiro, A. (2017). Between autonomy and control: Strategies of arbitrage in the "on-demand" economy. New Media \& Society. doi:10.1177/ 1461444817738236

Stark, D. (2009). The sense of dissonance: Accounts of worth in economic life. Princeton, NJ: Princeton University Press.

Uber. (2014). Your guide to the Uber rating system. Retrieved from https://www.dropbox.com/s/ b0gnhj14o226y6l/The\%20Rating\%20System.pdf

Uber Help. (2017). How to improve ratings. Retrieved from https://help.uber.com/h/f0934623-5fbc-46288dd0-565d5e451882

Uber Newsroom. (2017, March 15). A new navigation experience for drivers. Retrieved from https://www. uber.com/newsroom/a-new-navigation-experiencefor-drivers

van Doorn, N. (2017). Platform labor: On the gendered and racialized exploitation of low-income service work in the "on-demand" economy. Information, Communication and Society, 20(6), 898-914.

\section{About the Authors}

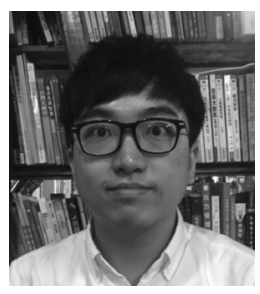

Ngai Keung Chan is a PhD student in the Department of Communication at Cornell University. His current research examines how data-driven technologies affect Uber drivers' work practices and emotional labor in the digital service economy. His research has appeared in Space and Culture, Computers in Human Behavior, and China Perspectives.

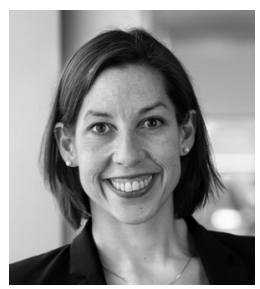

Lee Humphreys is an Associate Professor in Communication at Cornell University. She studies the social uses and perceived effects of communication technology, specifically focusing on mobile and social media. She is the author of The Qualified Self: Social Media and the Accounting of Everyday Life (MIT Press, 2018). Her research has appeared in Journal of Communication, New Media \& Society, and the Journal of Computer-Mediated Communication, among others. She received her PhD from the University of Pennsylvania's Annenberg School in 2007. 